Original Research Article

\title{
The prospective, non-interventional, pharmaco-vigilant study of antibiotic treatment of pediatric patients in pediatric intensive care unit and in-patient wards suffering from sepsis in a tertiary care hospital
}

\author{
Subhradipta Bhattacharyya ${ }^{1 *}$, Manjushree Mohanty ${ }^{2}$
}

\begin{abstract}
${ }^{1}$ Department of Pharmacology, MGM Medical College \& LSK Hospital, Kishanganj, Bihar, India

${ }^{2}$ Department of Pharmacology, KIMS, Bhubaneswar, Orissa, India
\end{abstract}

Received: 09 May 2018 Accepted: 04 June 2018

*Correspondence to: Dr. Subhradipta Bhattacharyya, Email:subhradiptabhattacharyya @yahoo.co.in

Copyright: (C) the author(s), publisher and licensee Medip Academy. This is an openaccess article distributed under the terms of the Creative Commons Attribution NonCommercial License, which permits unrestricted noncommercial use, distribution, and reproduction in any medium, provided the original work is properly cited.

\begin{abstract}
Background: Sepsis with septic shock has remained a dreadful disease inspite of early intervention mostly due to lack of sensitivity to first line antibiotics. This observational study was conducted to evaluate the utilization of antimicrobials and sensitivity-resistance pattern in paediatric patients suffering from sepsis due to various causes.
\end{abstract}

Methods: A prospective, non-interventional, pharmaco-vigilant study. 38 patients suffering from sepsis admitted in paediatric ward and PICU from September 2012 to February 2014 were evaluated. The research protocol was approved by Institutional Ethical Committee, KIMS. Data were collected from case sheets of patients from the ward as well as Medical Record and Data section. Average of Data on a continuous scale was expressed as a mean along with standard deviation, Categorical data was expressed as percentage. Comparative statistical analysis was done by using student's t-test in respect of data measured on a continuous scale. All differences with $\mathrm{P}$ value below 0.05 were labelled as statistically significant.

Results: Total 38 patients were admitted with provisional diagnosis of sepsis. $52.5 \%$ patients were suffering from $\geq 2$ diseases while pneumonia $(64 \%)$ and meningitis (22\%) were observed to cause sepsis. $10(27 \%)$ patients presented with septic shock while $39.3 \%$ patients having single organ failure and $10.4 \%$ patients having multiple organ failure. inj Dopamine (33\%) and inj Dobutamine 11\% were used and ventilator was used in 1 patient. Most of the patients received multiple antibiotics in I.V. route, $30(79 \%)$ patients received $\geq 3$ antibiotics. Linezolid+ piperacillin + tazo + aminoglycoside $+4^{\text {th }}$ drug combination was observed to be used in $34 \%$ patients while Linezolid $+3^{\text {rd }}$ gen. cephalosporin + aminoglycoside+ $4^{\text {th }}$ drug combination was used in $21 \%$ patients. Cephalosporin was most frequently withdrawn antibiotic (47\% of use). High resistance was observed for both gram +ve and gram -ve bacteria against cephalosporin.

Conclusions: Anti-MRSA agents (Linezolid) and anti-MDR GNB agent (Meropenem) significantly used to control the severe sepsis in conjunction to Piperacilin/ ceftriaxone + amikacin.

Keywords: Antibiotic resistance, Combination of antibiotic, Sepsis, Septic shock

\section{INTRODUCTION}

Sepsis is one of the leading cause of mortality and morbidity of pediatric population. ${ }^{1}$ Sepsis causes significant morbidity and mortality in children. Growing resistance against commonly used antibiotics has made the situation worse.

According to age, it can be categorised to neonatal sepsis (from birth to one month), Infants (1 month to 1 year), child (1 yr to $12 \mathrm{yr}$ ) and sepsis in adolescent (>12 years). 
Sepsis can be originated from various types of infection like bacterial, viral or fungal though no specific site of infection is found in most of the patients suffering from bacteremia or septicaemia. $^{2}$

However, certain diseases or disease like states like pneumonia, meningitis, peritonitis, UTI etc if inadequately treated produce sepsis. ${ }^{3}$ In significant number of patients more than one diseases co-exist (e.g., pneumonia with meningitis) in a patient. Single or Multiple organ-system failure sometimes observed in patients of severe sepsis with shock. Despite early hospitalisation with prompt antibiotic administration, high mortality rate is observed.

Ever-increasing resistance against commonly used antibiotics is responsible for treatment failure in most of the cases.

In this present work, it was tried to evaluate the patients from one month to 15 years of age admitted in PICU and Pediatric ward over a period of 18 months. The antibiotic utilization pattern was evaluated along with sensitivityresistance observed to antibiotics against isolated bacteria.

The observational study was conducted to evaluate the utilization of antimicrobials and sensitivity-resistance pattern in pediatric patients suffering from sepsis due to various causes.

\section{Plan of the study}

From September 2012 to February 2014 patients admitted in pediatric ward and PICU with provisional diagnosis of SEPSIS or BACTEREMIA were evaluated.

The present study is a non-interventional, uncontrolled, open chart, pharmaco-epidemiological and pharmacovigilant study. The research protocol was approved by Institutional Ethical Committee, KIMS.

\section{METHODS}

Within the study period from September 2012 to February 2014 about 70 patients were admitted in PICU and ward with proven or suspicion of sepsis of various infectious origin. Out of these patients, 38 patients have been evaluated. Data were collected from case sheets of patients from the ward as well as Medical Record and Data section of the Indoor patients receiving antibiotics along with supportive medications with provisional or confirmed BACTEREMIA OR SEPSIS.

\section{Inclusion criteria}

- $\quad$ Patients receiving antibiotics along with supportive medications with provisional or confirmed diagnosis of sepsis suffering from various infections like pneumonia, meningitis etc.
- $\quad$ Patients with confirmed diagnosis of sepsis who were discharged within one or two days, were excluded from the study.

\section{Exclusion criteria}

- $\quad$ Outdoor patients were excluded.

- Patients with severe renal impairment or hepatic failure were excluded from study sample.

- $\quad$ Patients who died were excluded from this study.

\section{Study of the drug utilization pattern}

Patients admitted in pediatric department were examined and data were collected according to pre-structured proforma. The following data were collected:

\section{Demographic profile}

This includes name, age, sex, body weight of the patients. These would help to illustrate the prevalence of a particular disease on different age or weight groups. As for example, Prevalence of sepsis, pneumonia or meningitis is significantly higher in 1m-1 yr age group (Figure 1).

\section{Clinical presentation}

This consists of history, brief clinical description, duration of illness, associated complaints, antibiotics taken elsewhere before admission in KIMS, previous illness or relevant family history etc.

\section{General and systemic examination}

It includes recording of heart rate, $\mathrm{BP}$, anemia, jaundice and edema and weight. Weight of the patient was required for the dosage calculation.

\section{Laboratory routine and specific investigation}

- Laboratory investigations like TLC, DLC, $\mathrm{Hb} \%$, reticulocyte count, platelet count, MP, LFT, serum urea, creatinine, uric acid etc. were done routinely.

Blood c/s, Urine c/s, sputum c/s etc. were done whenever required. ${ }^{4}$

- $\quad$ ABG (arterial blood gas analysis) were done in patients of sepsis from pneumonia with respiratory failure routinely.

- $\quad$ CSF gram stain, $\mathrm{C} / \mathrm{S}$ and cytology were done after LP routinely in Meningitis patients. CSF for viral marker or LAT (latex agglutination Test) to detect bacterial antigen were done in significant number of patients.

- $\quad$ Pleural fluid cytology or biochemical tests were done in patients of Pneumonia with pleural effusion.

- $\quad$ CRP, ALK-P, PT, serum electrolytes etc were done in sepsis patients. 
Other investigations

- CXR are done routinely in Pneumonia or LRTI patients and repeated during the course of treatment.

- CT- thorax, USG chest were done in complicated pneumonia patients to detect pleural effusion or pneumothorax.

- $\quad$ EEG were done when seizure develop in meningitis or sepsis patients.

- Fundoscopy was done before LP in meningitis patients to detect papilloedema due to increased ICP.

- In meningitis patients Audiometry in most of the patients and BERA (Brain-stem Evoked Auditory Response) in a few cases were done to detect sensorineural hearing impairment at the time of discharge from hospital.

\section{Drug utilization pattern}

Data about the drugs prescribed was collected, mostly the antibiotics and antimicrobials. The route of administration, the dose, the frequency of dosing and the duration etc were noted.

\section{Efficacy assessment of drugs}

\section{Priary efficacy parameters}

- The clinical status of the patients was evaluated at frequent interval, from admission to discharge. The clinical improvement or deterioration was assessed in terms of various signs and symptoms, like improvement of shock and acidosis, improvement in GCS score, remission of fever, normalization of respiratory rate and pulse rate, subsidence of neck rigidity in meningitis, improvement of $\mathrm{PAO} 2 / \mathrm{FiO} 2$ in ventilated patients of sepsis and pneumonia etc. ${ }^{5}$

- Clinical improvement is considered as the major determining factor to evaluate the efficacy of an antimicrobial along with the safety.

- Severe sepsis patients often develop septic shock with or without single or multiple organ failure. ${ }^{6,7}$ Cardiovascular failure or dysfunction, respiratory dysfunction, hematologic dysfunction, neurological dysfunction or hepatic dysfunction are developed either alone or two/more than two organ-system at a time. The data regarding organ failure or septic shock were recorded from clinical notes.

\section{Secondary efficacy parameters}

\section{Laboratory investigations}

- Normalization of blood count (TLC, DLC, platelet count), $\mathrm{Hb} \%$, BUN, serum electrolytes, CRP, Alk-P etc.

- CSF sterilization and normalization of cytology in meningitis, eradication of bacteria from blood c/s or urine $\mathrm{c} / \mathrm{s}$ in sepsis, sterilization of pleural fluid or sputum in LRTI or pneumonia etc.

\section{Radiological assessment}

Resolution of infiltration in CXR etc were noted in Pneumonia or LRTI, normalization of findings in CT/MRI in meningitis etc.

Adverse drug reaction monitoring.

\section{Statistical analysis}

Means of data on a continuous scale was expressed along with standard deviation /Error (Mean +/- SE). Comparative statistical analysis of two means was done by using Student's t-test. For qualitative data, Chi square test was applied for comparisons of two means. All differences with $\mathrm{P}$ value below 0.05 were labelled as statistically significant. ${ }^{8,9}$

\section{RESULTS}

\section{Patient particulars}

Among the 38 patients there were 25 male patients and 13 female patients.

Average age of the patients $=7.5 \pm 0.75(\mathrm{SE}) \mathrm{yr}$.

Average body weight $=23.58 \pm 2.17(\mathrm{SE}) \mathrm{Kg}$.

Average days of hospitalization $=14.4 \pm 1.17(\mathrm{SE})$ days .

\section{Age distribution of PICU patients}

- There were $18.4 \%$ of total patients are within the age group of $1 \mathrm{~m}-1 \mathrm{yr} .10 \%$ of total patients are within the age group of $1 \mathrm{yr}-5 \mathrm{yr}$ and $29 \%$ patients are within the age group of $5-10 \mathrm{yr}$. $40 \%$ patients are within the age group of 10yr-15 yr (Figure 1).

- There is significantly higher percentage of patient density (year wise) in the age group of $1 \mathrm{~m}-1 \mathrm{yr}$ (Chisquare applied, $\mathrm{p}<0.05)$.

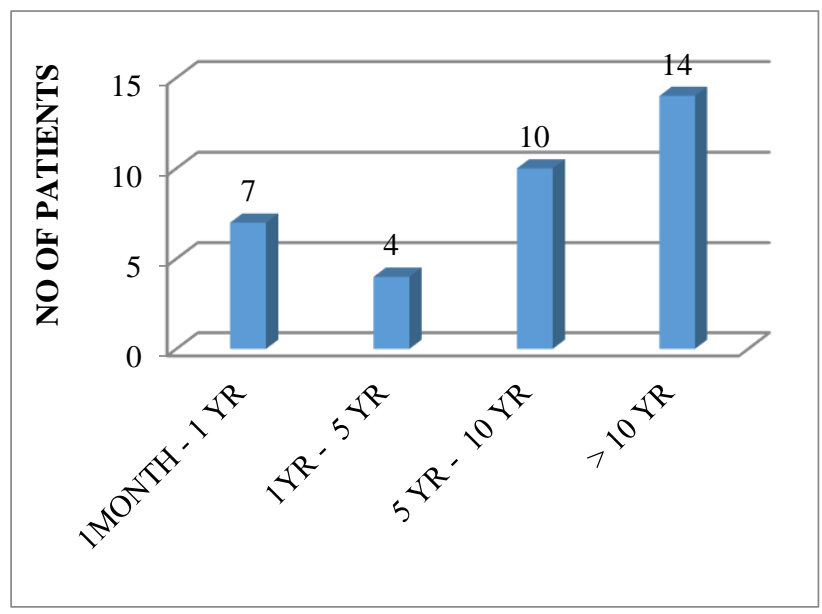

Figure 1: Age distribution of sepsis patients in PICU. 


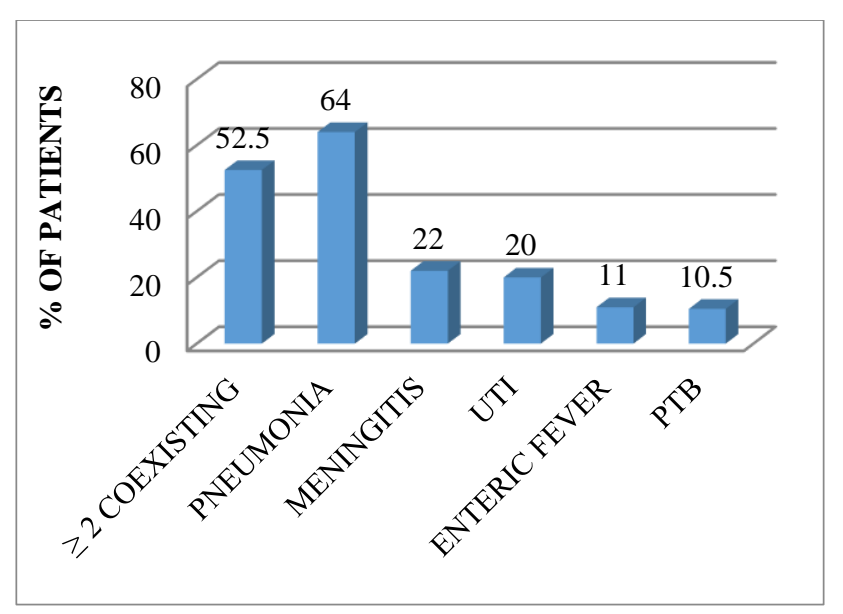

Figure 2: Diseases contributing sepsis.

\section{Diseases assosiated with sepsis}

Among the 38 patients evaluated, 10 (27\%) patients presented with septic shock.

- It was found that $39.3 \%$ have failure of one organ, $10.4 \%$ patients have multi-organ failure. However, $50 \%$ patients did not develop any organ failure.

- $\quad$ Pneumonia or LRTI (64\%) has been found to be most frequent disease to cause bacteremia or septicemia. In considerable number of patients pneumonia was co-existed with meningitis or UTI or bacillary dysentery (Figure 2).

- Meningitis (22\%) alone or co-existing with pneumonia or UTI etc. has been found to be second most frequent cause to develop septicemia. ${ }^{10}$

- In significant number of patients $(2.5 \%)$ viral or fungal infection had contributed to complicate the existing illness.

- In $52.5 \%$ patients two or more than two diseases (e.g; pneumonia with meningitis with/without age) were co-existing.

\section{Supportive treatments}

\section{Ionotropes}

Septic shock was initially treated with iv fluids. Inj dopamine $5-10 \mu \mathrm{g} / \mathrm{kg} / \mathrm{min}$ was most commonly used ionotropes. Inj dopamine was used in $33 \%$ cases. Inj dobutamine $(11 \%)$ was added in a few cases.

- Anticonvulsants: Sepsis patients often present with seizure and febrile convulsion. Anti-epileptic drugs were used in $5.4 \%$ cases.

- $\quad$ Steroids: Inj dexamethasone was used $11 \%$ patients.

- Inj calcium-gluconate: Inj Ca-gluconate was used in $8.3 \%$ cases.

- Ventilator: In 1 patient ventilator was used.

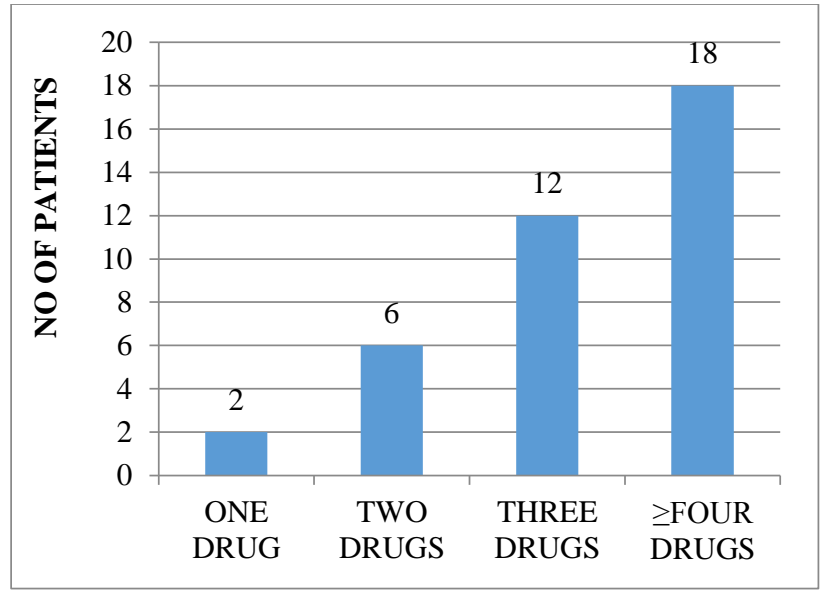

Figure 3: Different patients to receive multiple drugs.

As sepsis is an emergency condition most of the patients were immediately treated with combination of antibiotics in intravenous route along with supportive measures. ${ }^{11-13}$

- $\quad$ On an evaluation of empirical therapy, treatment was started with one drug regimen in $5(13.2 \%)$ though Out of 5 only two patients completed their treatment successfully with single antibiotic.

- In $21(55 \%)$ patients treatment were started with two antibiotics regimen. Only 6 out of 21 patients completed their treatment with having two drugs and in 15 patients $\geq 1$ drugs were added.

- In $11(29 \%)$ patients treatment were started with three drug regimen, only in 1 or 2 patients there was alteration/addition.

- $\quad$ There were $12(31.6 \%)$ patients were found to have three drugs; 30 (79\%) patients received three or more than three antibiotics during their illness whereas 18 (47.4\%) patients received $\geq$ four drugs (Figure 3 ).

- In $5(13.1 \%)$ patients treatment was started with one I.V. antibiotic however in three of them one or more antibiotics were added.

- $\quad$ In $12(31.5 \%)$ patients either one or more antibiotics were replaced by other after the empirical regimen was started (e.g., Inj Linezolid was added or Inj Ceftriaxone was replaced by Inj Piperacillin, etc.).

\section{Different combinations of antibiotics used}

Used Drug combinations can be enumerated as follows:

- Linezolid + cefotaxim/ ceftriaxone + amikacin/gentamicin $\pm 4^{\text {th }} \mathrm{drug}=21 \%$ patients

- $\quad$ Linezolid+ piperacillin + amikacin/gentamicin $\pm 4^{\text {th }}$ drug $=34 \%$

- Carbapenem+ beta lactam $+3^{\text {rd }}$ drug $=10.5 \%$

- Betalactam + aminoglycoside $+3^{\text {rd }}$ drug $=18 \%$

- $\quad$ Piperacillin \pm bli + aminoglycoside $=8.3 \%$

- Cephalosporin \pm bli + aminoglycoside $=5.5 \%$

- $\quad 3^{\text {rd }} \mathrm{drug}=\mathrm{tab}$ cefuroxime/ tab ofloxacin/ tab azithro; $4^{\text {th }}$ drug $=$ antifungal/ anti malarial/ ATT 


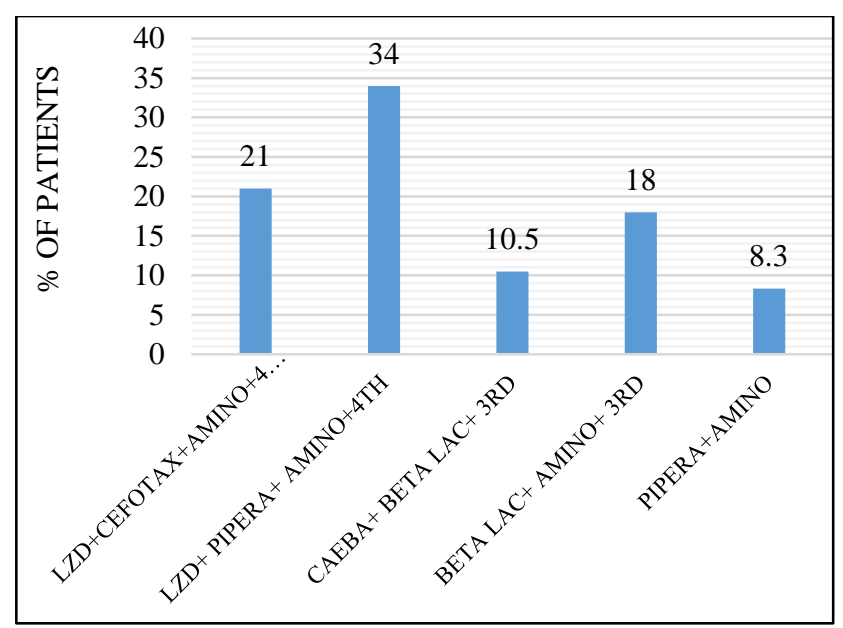

$3^{\text {rd }}=\mathrm{TAB}$ cefuroxime $/ \mathrm{TAB}$ oflox/ TAB azithro $4^{\mathrm{th}}=$ ATT $/$ anti fungal/ anti malarial

Figure 4: Different drug combinations.

- There is $79 \%$ of patients received three or more than three drugs.

- Among them $21 \%$ received linezolid + cephalosporin + aminoglycoside \pm 4 th drug combination. $34 \%$ patients received linezolid+ piperacillin+ aminoglycoside+ 4th drug combination. $10.5 \%$ patients received carbapenem+ $\beta$-lactam $+3^{\text {rd }}$ drug combination and $18.4 \%$ patients received $\beta$-lactam + aminoglycoside $+3^{\text {rd }}$ drug combination (Figure 4).

- In $11(28.9 \%)$ patients the existing empirical regimen was totally replaced by new drug regimen (e.g., Combination of Inj Pipzo and Inj Amikacin were replaced by Inj Linezolid and Inj Meropenem).

\section{Duration of use}

On average the different drugs were used are as following; Linezolid (10 days), Imipenem (9 days), Piperacillin-tazo (9 days), Cephalosporin (8 days), Amoxi-clav (7 days), Aminoglycoside (7 days) and Macrolide (3 days).

\section{Individual antibiotics}

- Aminoglycoside (22.4\% of total antibiotic use) and Cephalosporin (18\%) were two most frequently used drugs followed by Piperacillin+tazobactum (11.2\%), Quinolone (7.8\%), Amoxi-clav (5\%) and Macrolide $(3.9 \%)$.

\section{Use of cephalosporin}

- Ceftriaxone $\pm \beta$-LI was the most frequently used cephalosporin (87\%) (Figure 5).

- Use of cephalosporin was found to be limited within second and third generation. Fourth generation cephalosporin like Cefepime or Cefpirome were not used.

- Inj ceftriaxone \pm sulbactum was used in dose of $50 \mathrm{mg} / \mathrm{kg}$ I.V B.D.
- Inj cefoperazone \pm tazobactum was used in a dose of $50 \mathrm{mg} / \mathrm{kg}$ I.V B.D for moderate infection. For severe infection the dose was found to be $110 \mathrm{mg} / \mathrm{kg} \mathrm{I.V}$ B.D.

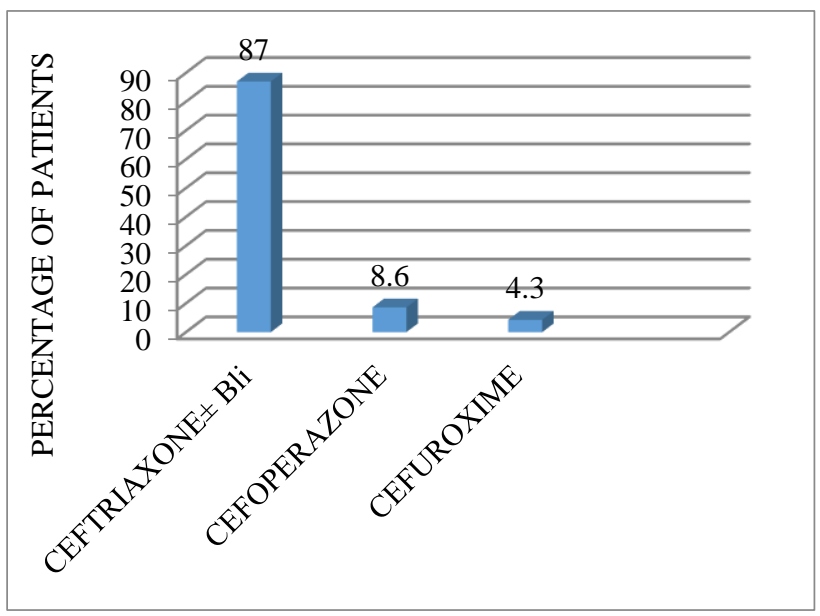

Figure 5: Use of different cephalosporins.

\section{Use of quinolone (7.8\%)}

Ofloxacin (70\%) and Ciprofloxacin (30\%) were the most frequently used quinolones.

\section{Use of macrolide (3.9\%)}

Azithromycin (87.5\%) and Clarithromycin (12.5\%) were mostly used. Azithromycin was used orally in a dose of $11.4 \mathrm{mg} / \mathrm{kg}$ OD. Clarithromycin was used orally in a dose of $9.3 \mathrm{mg} / \mathrm{kg} \mathrm{BD}$.

\section{Use of penicillin}

- $\quad$ Piperacillin $\pm \beta$ LI (57\%) was the most frequently used Penicillin followed by Ampicillin \pm cloxacillin (27\%) and Amoxicillin $\pm \beta$ LI.

- Piperacillin was used in the dose range of 75$120 \mathrm{mg} / \mathrm{kg}$ I.V TDS.

- Inj Ampicillin/amoxicillin + cloxacillin was used $40 \mathrm{mg} / \mathrm{kg} / \mathrm{dose}$ I.V QDS/TDS.

- In oral form Amoxi+ clav or Amoxicillin+ cloxacillin was used $20 \mathrm{mg} / \mathrm{kg} /$ dose TDS.

\section{Use of aminoglycosides (22.4\%)}

- Amikacin (82.5\%) was most commonly used followed by Gentamicin (7.5\%) and Netilmycin $(10 \%)$.

- Inj Amikacin was used in the dose range of 7.5$10 \mathrm{mg} / \mathrm{kg}$ I.V. BD. Inj Netilmycin was used $16 \mathrm{mg} / \mathrm{kg}$ I.V B.D. Inj Gentamicin was used $2.2 \mathrm{mg} / \mathrm{kg}$ I.V B.D.

\section{Use of anti-MRSA and carbapenem}

- Linezolid was given to total 21 patients. Among them in 5 patients it was given empirically at the beginning 
and in 16 patients it was added later when gram positive cocci like staph. hemolyticus were isolated (Figure 6).

- Inj Linezolid was used in a dose of $10 \mathrm{mg} / \mathrm{kg} / \mathrm{dose}$ I.V TDS/B.D.

- $\quad$ Once the patient was stabilized, oral formulations (tab/syrup) were used instead of I.V. in about 15 patients.

- In one patient Inj Linezolid was withdrawn due to thrombocytopenia.

- Inj Vancomycin was used in a dose of $10 \mathrm{mg} / \mathrm{kg} / \mathrm{dose}$ I.V. TDS/QDS in 2 patients.

- $\quad$ Meropenem was used in 7 patients. In 5 cases it was added later on. The dose was $20 \mathrm{mg} / \mathrm{kg}$ I.V. TDS or $50 \mathrm{mg} / \mathrm{kg}$ I.V BD.

- In four patients (10.5\%) both Linezolid and Meropenem were used. ${ }^{14}$ Among them in 2 patients both the drugs were started from beginning.

Antifungals were used in $4 \%$ patients.

\section{DISCUSSION}

\section{Alteration/addition or withdrawal of antibiotics}

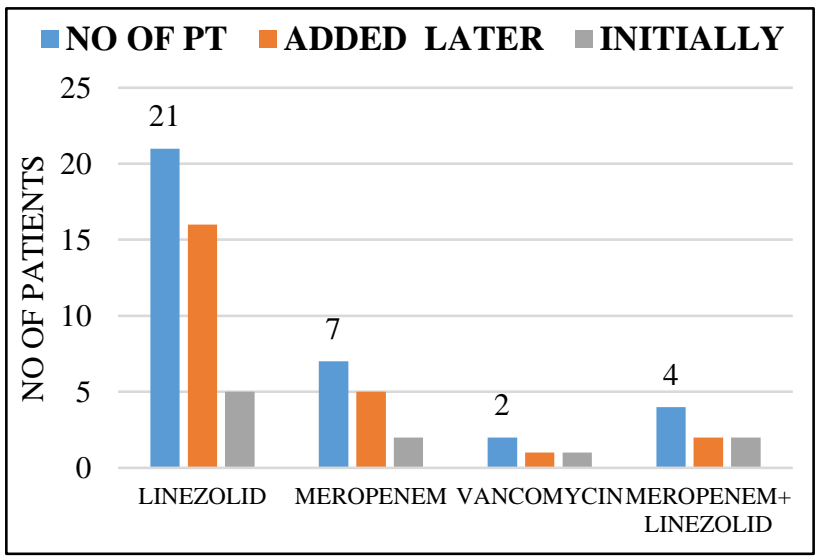

Figure 6: Use of linezolid and carbapenem.

- Among the first line antibiotics aminoglycoside was used in $26(68 \%)$ patients, cephalosporin $\pm \beta L I$ was used in $26(68 \%)$ patients, piperacillin $\pm \beta L I$ in 12 $(31.6 \%)$ and Amoxi-clav in $8(21 \%)$ patients.

- Cephalosporins were withdrawn in 11 patients (42.3\% withdrawal), aminoglycoside in 3 patients (11.5\% withdrawal), piperacillin $\pm \beta \mathrm{LI}$ in 2 patients (16.7\% withdrawal) and Amoxi-clav in 2 patients ( $25 \%$ withdrawal). Meropenem was continued in all the given patients (Figure 7).

- On applying chi square test significant variation in percentage of antibiotic withdrawal $(p<0.001)$ was observed which corroborated the findings in the sensitivity-resistance pattern found in the isolated bacteria.

- Among the second line drugs linezolid was used in $21(55 \%)$ patients, meropenem 7 (18.4\%), quinolone
$7(18.4 \%)$, Macrolide $4(10.4 \%)$ and vancomycin 2 (5.3\%) patients (Figure 8).

- $\quad$ Linezolid was withdrawn in 2 patients, vancomycin in 1 patient, quinolone in 2 patients.

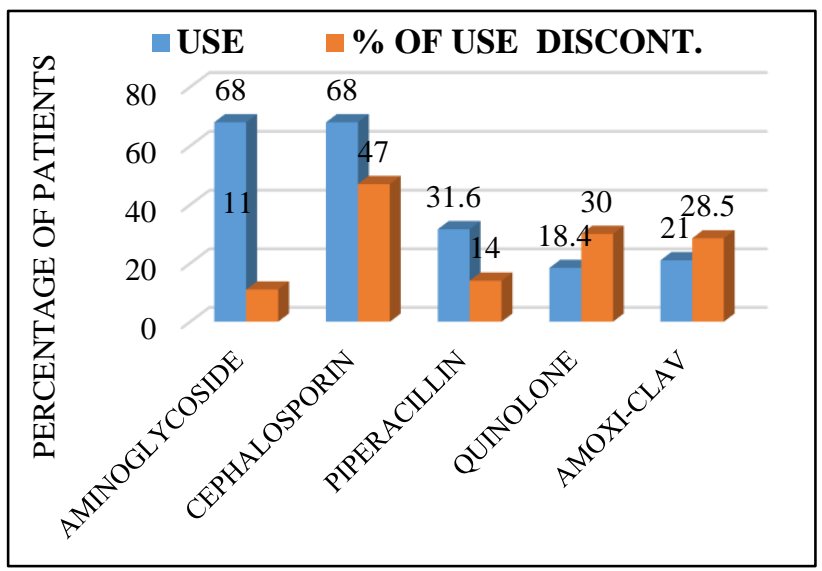

Figure 7: Use and withdrawal of antibiotics.

- When no bacteria was found but severity of infection was not decreased, inj linezolid/vancomycin for gram positive coverage and inj piperacillin /meropenem for gram negative coverage were administered simultaneously. ${ }^{15}$

- $\quad$ In 4 patients Linezolid and Meropenem both were used.

- Isolated gram +ve bacteria had shown high resistance against commonly used antibiotics.

- Poor sensitivity was observed for 3rd generation cephalosporin $\pm \beta \mathrm{LI}$ against gram positive $(5.5 \%)$ and gram -ve $(23.5 \%)$.

- Linezolid and vancomycin had shown $100 \%$ sensitivity to gram + ve bacteria.

- $\quad$ Effective sensitivity against gram -ve observed in carbapenem (72\%), aminoglycoside (64.7\%), piperacillin $\pm \beta \mathrm{LI}(57 \%)$ and Quinolone (58\%) (Figure 9).

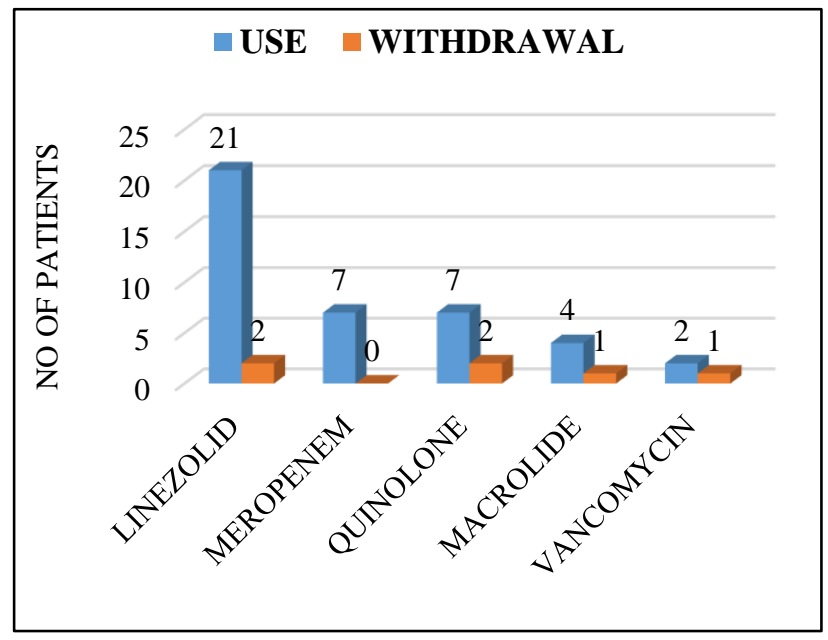

Figure 8: Use and withdrawal of anti-MRSA/ carbapenem. 


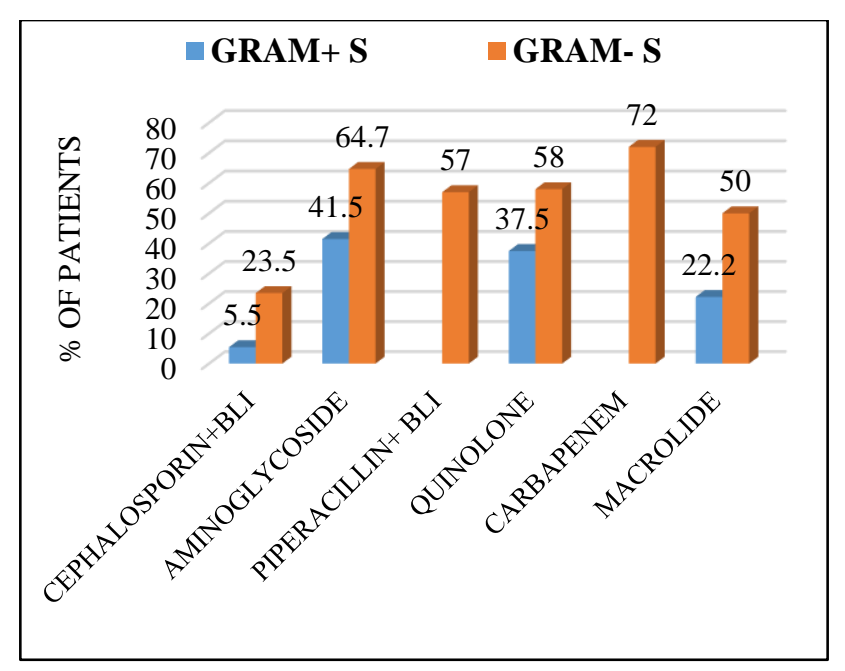

Figure 9: Sensitivity observed in isolated bacteria.

\section{Complications in sepsis patients in PICU and ward}

- The patients admitted in ward or PICU with provisional diagnosis of sepsis often present with fever or hypothermia, altered mental status, features of septic shock like increased CRT, non-measurable BP or non-palpable pulses etc.

- $\quad$ Among the 36 patients evaluated, 10 (27\%) patients presented with septic shock. ${ }^{6,7}$

\section{Severity of sepsis according to diseases}

It was observed that severity of sepsis was varied according to diseases of origin, however in most of the cases two or more than two diseases were (e.g., pneumonia with tuberculosis or meningitis with pneumonia etc) present in a patient. To receive more than three antibiotics and mean duration of hospitalisation were considered the parameters of severity of sepsis. The patients can be categorised according to diseases of origin in following groups:

- Sepsis predominantly originating from Pneumonia: $14(87.5 \%)$ patients received $\geq 3$ antibiotics, $9(56 \%)$ patients to receive $\geq 4$ antibiotics and mean duration of hospitalization was 12.5 \pm 5.34 days (Figure 10).

- $\quad$ Sepsis predominantly originated from Tuberculosis: $4(100 \%)$ patients received $\geq 3$ or 4 antibiotics (besides ATT) and mean duration of hospitalization was $28 \pm 5.83$ days.

- $\quad$ Sepsis predominantly originated from Meningitis: 2 (33\%) patients received $\geq 3$ or 4 antibiotics and mean duration of hospitalization was $9 \pm 3.11$ days.

- $\quad$ Sepsis with undiagnosed cause: 2 (50\%) patients received $\geq 3$ antibiotics, $1(25 \%)$ patients to receive $\geq 4$ antibiotics, and mean duration of hospitalization was $10 \pm 3.55$ days.

- Sepsis from viral/bacterial/protozoal infection: 7 (100\%) patients received $\geq 3$ antibiotics, 4 (57\%) patients to receive $\geq 4$ antibiotics and mean duration of hospitalization was $17 \pm 7.61$ days.

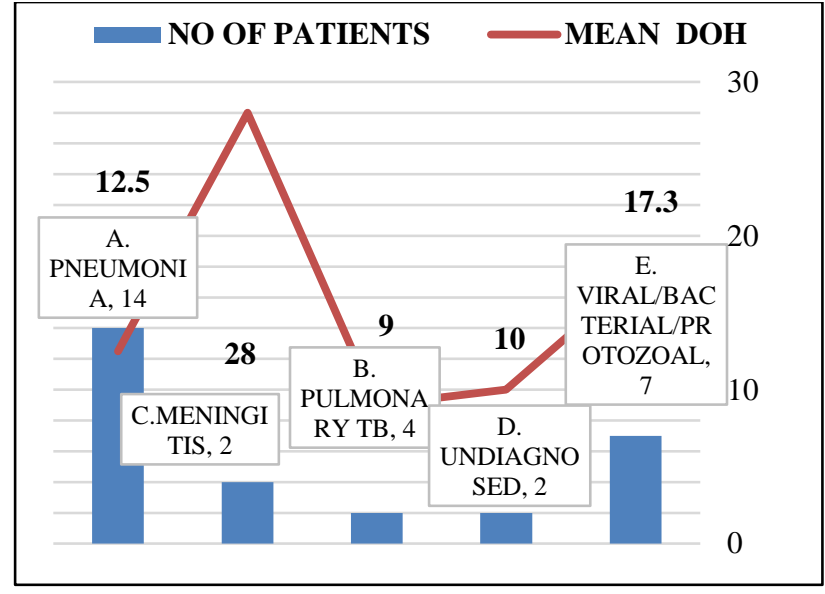

Figure 10: Number of patients (in different diseases) to receive $\geq 3$ antibiotics and mean duration of hospitalisation (days).

However, since the severity of sepsis among 20 patients was very high, four or more than four antibiotics were administered during their treatment period. It was found that $100 \%$ of patients of sepsis originating from Pulmonary tuberculosis, $56 \%$ patients with pneumonia and $57 \%$ patients of viral/bacterial/protozoal infection required $\geq 4$ antibiotics (Figure 11).

Applying chi-square test it was found $p<0.001$ i.e. the variation of severity of sepsis among different groups were due to type and severity of diseases/infections not by chance.

Patients with severe or very severe sepsis were deliberately included in the study that masks the actual scenario of sepsis in PICU and pediatric wards. For categorization of sepsis patients into moderate/severe or very severe, clinical notes from the case sheets were entirely followed.

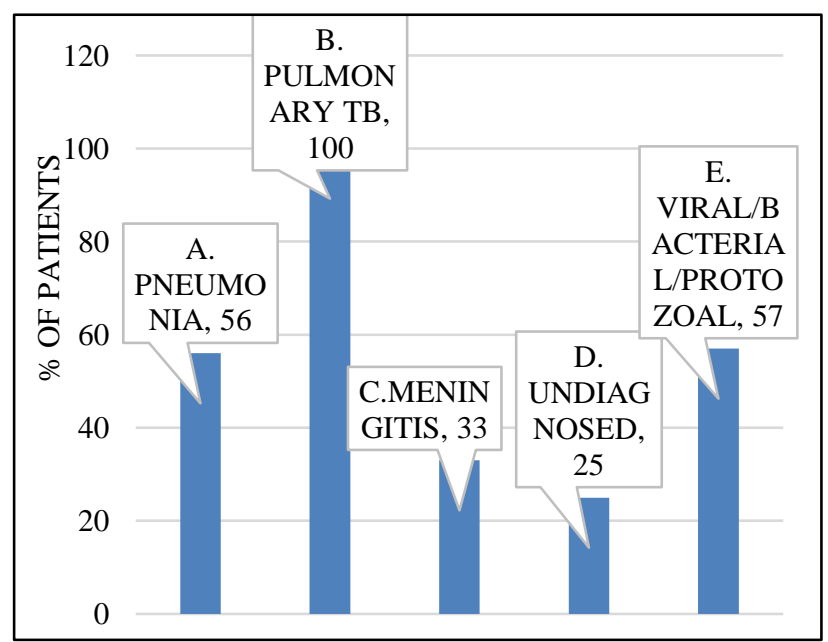

Figure 11: Percentage of patients of different diseases to receive $\geq 4$ antibiotics.

No sepsis severity scale like, The Pediatric Logistic Organ Dysfunction (PELOD) score or The Pediatric multiple 
organ dysfunction score (P MODS) were not applied. Expired patients were excluded from the study. Mortality rate was not evaluated for determining the efficacy of antibiotics.

\section{CONCLUSION}

Poor sensitivity was observed for $3^{\text {rd }}$ generation cephalosporin $\pm \beta \mathrm{LI}$ against gram positive $(5.5 \%)$ and gram -ve $(23.5 \%)$. Effective sensitivity against gram -ve observed in carbapenem (72\%), aminoglycoside (64.7\%), piperacillin $\pm \beta$ LI $(57 \%)$ and Quinolone $(58 \%)$.

Linezolid and vancomycin had shown $100 \%$ sensitivity to gram +ve bacteria. Therefore, Anti-MRSA agents (Linezolid) and anti-MDR GNB agent (Meropenem) were used a lot confidently by the physicians to control the severe sepsis in conjunction with Piperacillin/ ceftriaxone + amikacin. 20 (52.6\%) patients with very severe sepsis were administered four or more than four antibiotics in combination which immensely improved the survival of the patients.

\section{ACKNOWLEDGEMENTS}

Authors are Grateful to Department of Microbiology and Dr. A K Mohanty (Professor and HOD Department of Pediatrics) KIMS, Bhubaneswar, Orissa.

They are also grateful to Dr T.K. Bhattacharyya, Professor, Department of Pharmacology, MGM Medical College, Kishanganj, Bihar.

Funding: No funding sources Conflict of interest: None declared

Ethical approval: The study was approved by the Institutional Ethics Committee of KIMS, Bhubaneswar, Orissa, India

\section{REFERENCES}

1. Khilnani P, Singhi S, Lodha R, Santhanam I, Sachdev A, Chugh K, et al. Pediatric Sepsis Guidelines: Summary for resource-limited countries. Indian journal of critical care medicine: peer-reviewed, official publication of Indian Society of Critical Care Medicine. 2010 Jan;14(1):41.

2. Camussi G, Tetta C, Ronco C, Fonsato V. Recent insights into the pathogenesis of severe sepsis. Critical Care and Resuscitation. 2005 Mar;7(1):32.

3. Dremsizov T, Clermont G, Kellum JA, Kalassian KG, Fine MJ, Angus DC. Severe sepsis in communityacquired pneumonia: when does it happen, and do systemic inflammatory response syndrome criteria help predict course?. Chest. 2006 Apr 1;129(4):96878.

4. Seifert H. The clinical importance of microbiological findings in the diagnosis and management of bloodstream infections. Clinical Infectious Diseases. 2009 May 15;48(Supplement_4):S238-45.

5. Seifert H. The clinical importance of microbiological findings in the diagnosis and management of bloodstream infections. Clinical Infectious Diseases. 2009 May 15;48(Supplement_4):S238-45.

6. Lorry R. Kache FS. Shock (Severe sepsis and septic shock) Kliegman: Nelson Textbook of Pediatrics. Part XVII-XXXIII, chapter 68,19 $9^{\text {th }}$ Edn; 2008.

7. Munford RS. Severe Sepsis and Septic shock, Harrison's Principle of Internal Medicine. $19^{\text {th }}$ Edition. 1751-1758.

8. Bernard Rosner. Fundamental of Biostatistics. Chapter 7 and chapter $8.7^{\text {th }}$ Edition.

9. Mahajan BK. Methods in Biostatistics. Chapter 9. $7^{\text {th }}$ Edition.

10. Cha'vez-Bueno S, McCracken GH. Bacterial Meningitis in Children Pediatr Clin N Am. 2005;52:795-810.

11. Kumar A, Zarychanski R, Light B, Parrillo JE, Maki D, Simon D, et al. Early combination antibiotic therapy yields improved survival compared to monotherapy in septic shock: A propensity-matched analysis. Crit Care Med. 2010:38.

12. Kumar A, Safdar N, Kethireddy S, Chateau D. A survival benefit of combination antibiotic therapy for serious infections associated with sepsis and septic shock is contingent on the risk of death: A metaanalytic/meta-regression study. Crit Care Med. 2010;38:8.

13. Safdar N, Handelsman J, Maki DG. Does combination antimicrobial therapy reduce mortality in Gramnegative bacteraemia? A meta-analysis. The Lancet infectious diseases. 2004 Aug 1;4(8):519-27.

14. Kaul DR, Collins CD, Hyzy RC. New developments in antimicrobial use in sepsis. Current pharmaceutical design. 2008 Jul 1;14(19):1912-20.

15. Sick AC, Tschudin-Sutter S, Turnbull AE, Weissman SJ, Tamma PD. Empiric combination therapy for gram-negative bacteremia. Pediatrics. 2014 Apr 1:peds-2013.

Cite this article as: Bhattacharyya S, Mohanty M. The prospective, non-interventional, pharmacovigilant study of antibiotic treatment of pediatric patients in pediatric intensive care unit and in-patient wards suffering from sepsis in a tertiary care hospital. Int J Basic Clin Pharmacol 2018;7:1529-36. 